\title{
Impact of Caspase-8 (CASP8) -652 6N Del and D302H Polymorphisms on Prostate Cancer in Different Ethnic Groups
}

\author{
Cheng-Dong Zhang ${ }^{1,3 \&}$, Hong-Tao Li ${ }^{1 \&}$, Kun Liu ${ }^{2 \&}$, Zhi-Di Lin³, Qi-Liu Peng ${ }^{5}$, \\ Xue Qin ${ }^{5}$, Min $\mathrm{He}^{1}$, Hua Wu${ }^{1}$, Zeng-Nan $\mathrm{Mo}^{4}$, Xiao-Li Yang ${ }^{1,3 *}$
}

\begin{abstract}
Background: Despite evidence suggesting roles for caspase-8 (CASP8) -652 6N del and D302H polymorphisms in prostate cancer (PCa), the association of these polymorphisms with PCa risk remains inconclusive. Therefore, a meta-analysis was performed to more precisely estimate the association of CASP8 -652 6N del and D302H polymorphisms with PCa susceptibility. Materials and Methods: A comprehensive literature search was conducted to identify all case-control studies of CASP8 D302H and $-652 \mathrm{6N}$ del polymorphisms and PCa risk. Odds ratios (ORs) and $95 \%$ confidence intervals (CIs) were used to assess the strength of the association and the precision of the estimate, respectively. Results: Nine -625 6N del studies and 4 D302H studies were included. CASP8 -652 6N del and D302H polymorphisms were not significantly associated with PCa risk in the overall analyses. However, in the subgroup analysis stratified by ethnicity, -625 6N del was significantly associated with PCa risk in the East Asian and Indian populations under the recessive model. Furthermore, the subgroup analysis strongly suggested that D302H was associated with lower PCa risk in the Non-Indian population under the dominant model. Conclusions: In our meta-analysis, ethnic-specific differences were evident in the association of CASP8 $-6256 \mathrm{~N}$ del and D302H polymorphisms with PCa risk.
\end{abstract}

Keywords: Prostate carcinoma - CASP8 - SNPs - meta-analysis - ethnic groups

Asian Pac J Cancer Prev, 15 (18), 7713-7718

\section{Introduction}

Prostate cancer $(\mathrm{PCa})$ is the second most common cause of cancer death among men in the United States. According to the cancer statistics report from the American Cancer Society, over 241,000 men were diagnosed with PCa and approximately 28,000 died from the disease in 2012 (Siegel et al., 2012). It is well known that multiple risk factors are associated with $\mathrm{PCa}$, including advanced age, ethnicity, family history, and genetic variations. Currently, prostate-specific antigen (PSA)-based screening and its derivative measurements are the most common methods used to detect PCa. However, elevations in PSA can result from urinary tract manipulation (e.g., prostate biopsy, catheterization) and non-malignant conditions (e.g., benign prostatic hyperplasia or prostatitis) (Klein and Lowe, 1997). Therefore, traditional PSA-based screening lacks sufficient sensitivity and specificity to be considered ideal for PCa detection. Recent research has focused on identifying genetic polymorphisms associated with PCa risk, with the aim of improving early diagnosis, screening, and individualized chemotherapy. The number of single nucleotide polymorphisms (SNPs) associated with PCa susceptibility has been rapidly increasing (Zheng et al., 2008), and SNPs associated with tumor cell apoptosis have received the most attention. Caspases, a family of proteases, mediate the regulation of apoptosis. One of the initiator caspases, CASP8 (also known as FLICE), has been reported to play an important role in apoptosis (Ho and Hawkins, 2005). Given the role of CASP8 in apoptosis, genetic variants of CASP8 that can alter its function are likely to affect apoptosis in cancer cells.

Recently, many studies have reported that 2 common SNPs within the CASP8 promoter gene, $-6526 \mathrm{~N}$ del and $\mathrm{D} 302 \mathrm{H}$, are associated with many cancer types. The $-6526 \mathrm{~N}$ del polymorphism (rs3834129) is a 6-nucleotide insertion-deletion substitution at nucleotides 657_652. The D302H polymorphism (rs 1045485) is a G-C substitution at $1207 \mathrm{bp}$, which results in an aspartic acid to histidine exchange at position 302 in the amino acid sequence. Sun et al. (2007) first reported that the $-6526 \mathrm{~N}$ del variant reduced the risk of developing many types of cancer including lung, esophageal, gastric, colorectal, cervical, and breast cancers (Sun et al., 2007). However, in subsequent studies, demonstration of the protective effect of $-6526 \mathrm{~N}$ del failed to be replicated in several cancer

\footnotetext{
${ }^{1}$ Medical Scientific Research Center, Guangxi Medical University, ${ }^{4}$ Department of Urology, ${ }^{5}$ Department of Clinical Laboratory, First Affiliated Hospital of Guangxi Medical University, Nanning, ${ }^{3}$ Department of Urology, Affiliated Hospital of Youjiang Medical College for Nationalities, Baise, Guangxi Zhuang Autonomous Region, ${ }^{2}$ Department of Gastroenterology, The Third Hospital of Nanchang City, Nanchang, Jiangxi, China \&Equal contributors *For correspondence: cncsyxl@126.com
} 
types, including breast and prostate tumors (Cybulski. et al., 2007; Haiman et al., 2008). Interestingly, the variant genotype (del/del) of $-6526 \mathrm{~N}$ del exhibited a trend of increased PCa risk in a North Indian population (George et al., 2010; Kesarwani et al., 2011). In contrast, the same variant was highly associated with reduced PCa risk in a Chinese population (Fu et al., 2011). In contrast to $-6526 \mathrm{~N}$ del, the D302H H variant has demonstrated a consistent protective effect for breast carcinoma risk (Sergentanis and Economopoulos, 2010). However, the association of D302H with PCa risk has been inconclusive. Lubahn et al. and Meyer et al. reported that the $\mathrm{D} 302 \mathrm{H}$ polymorphism may play a protective role against PCa (Lubahn et al., 2010; Meyer et al., 2013); whereas, in a North Indian population, the $\mathrm{H}$ variant allele was associated with increased PCa risk (George et al., 2010).

Inconsistencies in the association of CASP8 polymorphisms with $\mathrm{PCa}$ risk may be attributable to different ethnicities. Therefore, we performed a metaanalysis to further evaluate the association of D302H and $-6526 \mathrm{~N}$ del with PCa risk.

\section{Materials and Methods}

\section{Literature search}

We performed a literature search using the following keywords "CASP8 [MeSH]", "prostate cancer [MeSH]", and "polymorphism" or "variation" or "mutation" in PubMed, Cochrane Library, and Web of Science databases. All eligible studies were published before July 1st, 2014. The reference lists of review and retrieved articles were hand searched at the same time. Abstracts, unpublished reports, and articles written in non-English languages were not considered. When overlapping data of the same patient population were included in more than 1 publication (sending email to the communication author to confirm this), only the most recent or complete study was used in the meta-analysis.

\section{Inclusion and exclusion criteria}

The following inclusion criteria were used to select literature for the meta-analysis:

(1) information on the evaluation of CASP8 -652 $6 \mathrm{~N}$ del or CASP8 D302H polymorphisms and PCa susceptibility; (2) case-control studies; and (3) studies with sufficient genotype data to calculate odd ratios (ORs) with $95 \%$ confidence intervals (CIs). Major criteria for study exclusion were: (1) no controls; (2) reviews and duplication of the previous publication; and (3) no usable data reported.

\section{Data extraction}

Two of the authors independently extracted the study information from all eligible publications based on the inclusion criteria above. Any discrepancies regarding study inclusion were resolved by discussion, and a third party was involved when necessary. The following characteristics were recorded from each study: the name of the first author, publication year, ethnicity of the population, and genotype frequencies of $-6526 \mathrm{~N} \mathrm{del}$ and D302H polymorphisms in both PCa cases and controls.
Ethnic descent was categorized into the following groups: Caucasian, Indian, East Asian, African, and other. For case-control studies, data were extracted separately for each group whenever possible.

\section{Statistical analysis}

Crude ORs with the corresponding 95\% CIs were used to detect the strength of the association of $-6526 \mathrm{~N} \mathrm{del}$ and D302H polymorphisms with PCa risk. For both CASP8 polymorphisms, the relationship between genotypes and PCa susceptibility was evaluated using co-dominant, recessive, over-dominant, and dominant genetic models. The relationship between alleles and PCa susceptibility was also examined using an allelic model. A Z-test was used to determine the significance of the overall OR, and $P<0.05$ was considered statistically significant. Betweenstudy heterogeneity and between-study inconsistency were evaluated using Cochran's Q statistic and estimating $\mathrm{I}^{2}$, respectively (Higgins et al., 2003). $P<0.10$ was considered statistically significant heterogeneity, and $\mathrm{I}^{2}$ was used to qualify variation in OR attributable to heterogeneity. In studies without substantial heterogeneity (Q-test $P \geq 0.10$ or $\mathrm{I}^{2}<50 \%$ ), the fixed effects model (Mantel-Haenszel method) was used to estimate the overall OR (Mantel and Haenszel, 1959). The random effects model (DerSimonian and Laird method) was used in studies with substantial heterogeneity (DerSimonian and Laird, 1986). In addition to the comparison among all subjects, stratification analyses by ethnicity were performed to explore the reasons for heterogeneity. To adjust for multiple comparisons, we applied the Benjamini-Hochberg (BH) method (Benjamini and Hochberg, 1995) and stepdown Bonferroni method (Holm, 1979), which control for false discovery rate (FDR) and familywise error rate (FWE), respectively. One-way sensitivity analyses were performed to assess the stability of the results. To investigate the potential publication bias, both visual funnel plot and Egger's linear regression test were applied, and $P<0.05$ was considered statistically significant. All statistical analyses were performed mainly using STATA version 12.0 (Stata Corp, College Station, TX, USA) and R package version 3.1.0.

\section{Results}

\section{Eligible studies}

Based on our inclusion criteria, 13 eligible independent studies in 7 reports were identified. Three of the reports contained data on different ethnic groups or variants (Haiman et al., 2008; George et al., 2010; Lubahn et al., 2010). Thirteen available studies with 5423 cases and 6994 controls were finally included in the meta-analysis. Among these 13 studies, 9 studies were eligible for the $-6526 \mathrm{~N}$ del polymorphism (3955 PCa cases, 4241 controls) and 4 studies were eligible for the $\mathrm{D} 302 \mathrm{H}$ polymorphism (1468 PCa cases, 2753 controls). The characteristics of the eligible studies are summarized in Table 1 and Table 2 , respectively.

\section{Meta-analyses}

In the overall analysis, the $-6526 \mathrm{~N}$ del polymorphism 
Table 1. Characteristics of Eligible Studies For -652 6N Del

\begin{tabular}{|c|c|c|c|c|c|c|c|c|c|c|c|c|}
\hline \multirow{2}{*}{$\begin{array}{l}\text { Author, } \\
\text { published year } \\
\text { Country }\end{array}$} & \multirow[t]{2}{*}{ Ethnicity } & \multirow{2}{*}{$\begin{array}{l}\text { Matching } \\
\text { criteria }\end{array}$} & \multirow{2}{*}{$\begin{array}{l}\text { Source } \\
\text { of control }\end{array}$} & \multirow{2}{*}{\multicolumn{2}{|c|}{$\begin{array}{l}\text { Genotyping No. of } \\
\text { methods Cases/Controls }\end{array}$}} & \multicolumn{4}{|c|}{ Cases } & \multicolumn{2}{|c|}{ Controls } & \multirow[t]{2}{*}{ HWE } \\
\hline & & & & & & ins/ins & ins/del & del/del & ins/ins & ins/del & $\mathrm{del} / \mathrm{del}$ & \\
\hline \multicolumn{13}{|l|}{ Cybulski $^{6}, 2007$} \\
\hline $\begin{array}{l}\text { Poland } \\
\text { Haiman }^{7}, 2008\end{array}$ & Caucasian & Region & PB & PCR-ASA & $485 / 965$ & 139 & 236 & 110 & 274 & 499 & 192 & Yes \\
\hline $\begin{array}{l}\text { USA } \\
\text { Haiman }^{7}, 2008\end{array}$ & Other & Age,BMI,smoking & $\mathrm{PB}$ & TaqMan & $852 / 616$ & 175 & 437 & 240 & 127 & 308 & 181 & Yes \\
\hline $\begin{array}{l}\text { USA } \\
\text { Haiman }^{7}, 2008\end{array}$ & East Asian & Age,BMI,smoking & PB & TaqMan & $707 / 709$ & 497 & 194 & 16 & 502 & 187 & 20 & Yes \\
\hline $\begin{array}{l}\text { USA } \\
\text { Haiman }^{7}, 2008\end{array}$ & Other & Age,BMI,smoking & $\mathrm{PB}$ & TaqMan & $110 / 111$ & 35 & 59 & 16 & 27 & 58 & 26 & Yes \\
\hline $\begin{array}{l}\text { USA } \\
\text { Haiman }^{7}, 2008\end{array}$ & Caucasian & Age,BMI,smoking & $\mathrm{PB}$ & TaqMan & $617 / 607$ & 246 & 275 & 96 & 257 & 269 & 81 & yes \\
\hline $\begin{array}{c}\text { USA } \\
\text { George }^{8}, 2010\end{array}$ & Caucasian & Age,BMI,smoking & PB & TaqMan & $443 / 422$ & 121 & 244 & 78 & 108 & 210 & 104 & Yes \\
\hline $\begin{array}{l}\text { India } \\
\text { Kesarwani }^{9}, 20\end{array}$ & Indian & Age,smoking & $\mathrm{PB}$ & PCR-RFLP & $165 / 208$ & 84 & 69 & 12 & 116 & 83 & 6 & Yes \\
\hline $\begin{array}{l}\text { India } \\
\mathrm{Fu}^{10}, 2011\end{array}$ & Indian & Age,smoking & HB & PCR-RFLP & $170 / 198$ & 86 & 72 & 12 & 109 & 83 & 6 & Yes \\
\hline China & East Asian & Age,smoking & HB & PCR-RFLP & $406 / 408$ & 257 & 132 & 17 & 211 & 159 & 38 & Yes \\
\hline
\end{tabular}

PCR-ASA, Allele-Specific Amplification; PCR-RFLP, polymerase chain reaction-restriction fragment length polymorphism; BMI, body mass index; HB, hospital based; PB, population based; HWE, Hardy-Weinberg equilibrium; Other, Hawaii, African

Table 2. Characteristics of Eligible Studies For D302H

\begin{tabular}{|c|c|c|c|c|c|c|c|c|c|c|c|c|c|}
\hline \multirow{2}{*}{$\begin{array}{l}\text { Author, } \\
\text { published year }\end{array}$} & \multirow[t]{2}{*}{ Country } & \multirow[t]{2}{*}{ Ethnicity } & \multirow{2}{*}{$\begin{array}{l}\text { Matching } \\
\text { criteria }\end{array}$} & \multirow{2}{*}{\multicolumn{2}{|c|}{$\begin{array}{l}\text { Source Genotyping } \\
\text { of control methods }\end{array}$}} & \multirow{2}{*}{$\begin{array}{c}\text { No. of } \\
\text { Cases/Controls }\end{array}$} & \multicolumn{4}{|c|}{ Cases } & \multicolumn{3}{|c|}{ Controls HWE } \\
\hline & & & & & & & DD & $\mathrm{DH}$ & $\mathrm{HH}$ & DD & $\mathrm{DH}$ & $\mathrm{HH}$ & \\
\hline George $^{8}, 2010$ & India & Indian & Age,smoking & PB & PCR-PIRA & $165 / 205$ & 111 & 48 & 6 & 155 & 46 & 4 & Yes \\
\hline Lubahn $^{12}, 2010$ & USA & Non-Indian & Age, PSA & PB & Direct Sequencing & $150 / 359$ & 137 & 11 & 2 & 305 & 52 & 2 & Yes \\
\hline Lubahn $^{12}, 2010$ & USA & Non-Indian & Age, PSA & PB & Direct Sequencing & $646 / 1701$ & 524 & 117 & 5 & 1270 & 401 & 30 & Yes \\
\hline Meyer $^{13}, 2010$ & Germany & Non-Indian & Age, PSA & $\mathrm{HB}$ & TaqMan & $507 / 488$ & 9 & 101 & 397 & 4 & 123 & 361 & Yes \\
\hline
\end{tabular}

PCR-RFLP, primer-introduced restriction analysis; PSA, prostate antigen; HB, hospital based; PB, population based; HWE, Hardy-Weinberg equilibrium

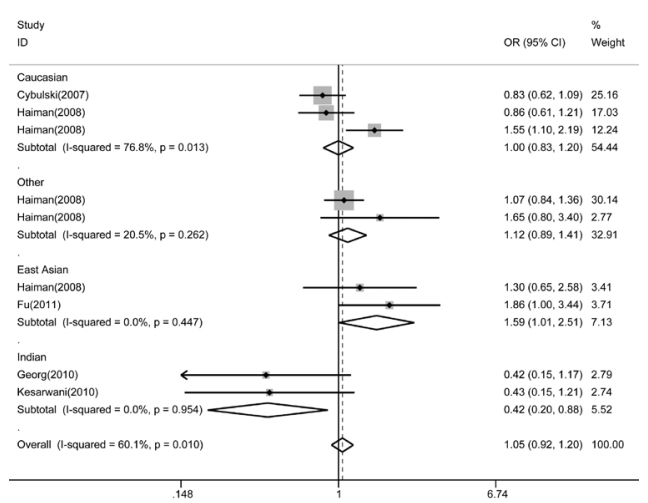

Figure 1. Forest Plot of Odd Ratios (ORs) of -652 6N Del Polymorphism Heterogeneous Co-Dominant Model (ins/del versus del/del) Associated with PCa Stratified by Ethnicity Using a Fixed-Effect Mode

was not significantly associated with $\mathrm{PCa}$ risk in the dominant (ins/del+del/del vs. ins/ins), recessive (del/del vs. ins/ins+ins/del), heterogeneous co-dominant (ins/del $v s$. del/del), or over-dominant (ins/ins+del/del vs. ins/del) genetic models. Interestingly, in the stratified analysis by ethnicity, the ins/del versus del/del showed different effects among the Indian $(\mathrm{OR}=0.42 ; 95 \% \mathrm{CI}=0.20$ $\left.0.88 ; p=0.021 ; P_{b o n}=0.063 ; P_{f d r}=0.042\right)$, East Asian (OR=1.59; 95\% CI=1.01-2.51;P=0.047; $P_{\text {bon }}=0.144$; $\left.P_{f d r}=0.094\right)$ and Caucasian $(\mathrm{OR}=1.03 ; 95 \% \mathrm{CI}=0.70-1.51$; $P=0.899 ; P_{b o n}=1 ; P_{f d r}=0.919 ;$ Figure 1) populations in the heterogeneous co-dominant model. Further investigation using the recessive model showed a significant positive

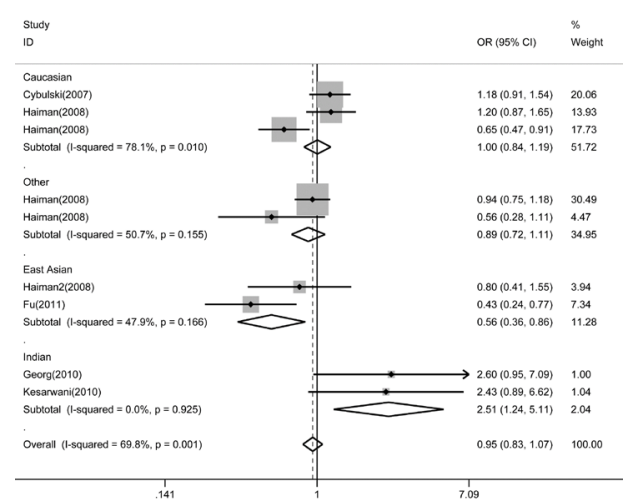

Figure 2. Forest Plot of Odd Ratios (ORs) of -652 6N Del Polymorphism Recessive Model (del/del vs. ins/ins+ins/del) Associated with PCa Stratified by Ethnicity Using a Fixed-Effect Mode

association of the $-6526 \mathrm{~N}$ del polymorphism with $\mathrm{PCa}$ risk in the Indian population $(\mathrm{OR}=2.51 ; 95 \% \mathrm{CI}=1.24$ $\left.5.11 ; P=0.001 ; P_{b o n}=0.044 ; P_{f d r}=0.042\right)$, whereas the -652 $6 \mathrm{~N}$ del polymorphism was negatively associated with $\mathrm{PCa}$ risk in the East Asian population $(\mathrm{OR}=0.56 ; 95 \%$ $\mathrm{CI}=0.36-0.86 ; P=0.008 ; P_{b o n}=0.032 ; P_{f d r}=0.032$; Figure 2 and Table 3 ).

The pooled meta-analysis results for the D302H polymorphism were similar to those for the -652 $6 \mathrm{~N}$ del polymorphism; the $\mathrm{D} 302 \mathrm{H}$ polymorphism was not significantly associated with $\mathrm{PCa}$ risk in the dominant $(\mathrm{DH}+\mathrm{HH} v s$. $\mathrm{DD})$, recessive (DD $v s$. $\mathrm{HH}+\mathrm{DH}$ ), heterogeneous co-dominant (DH vs. DD), or 
Cheng-Dong Zhang et al

Table 3. Meta-Analysis of the CASP8 -652 6N del Gene Polymorphisms On PCa Risk

\begin{tabular}{|c|c|c|c|c|c|c|c|c|c|}
\hline \multirow[t]{2}{*}{ Comparison } & \multirow[t]{2}{*}{ Population } & \multicolumn{5}{|c|}{ Test of association } & \multirow[t]{2}{*}{ Mode } & \multirow{2}{*}{$\begin{array}{c}\text { Egger's test } \\
P\end{array}$} & \multirow{2}{*}{$\begin{array}{l}\text { Heterogeneity } \\
\qquad I^{2}\end{array}$} \\
\hline & & OR & $95 \% \mathrm{CI}$ & $P$ & $P(\mathrm{BON})$ & $P(\mathrm{FDR})$ & & & \\
\hline \multirow[t]{4}{*}{ (ins/del+del/del) vs. ins/ins } & Overall & 0.97 & $0.88-1.07$ & 0.514 & 1 & 0.824 & $\mathrm{~F}$ & 0.785 & $47.70 \%$ \\
\hline & Indian & 1.23 & $0.92-1.64$ & 0.169 & 0.338 & 0.225 & $\mathrm{~F}$ & - & $0.00 \%$ \\
\hline & East Asian & 0.8 & $0.49-1.31$ & 0.381 & 0.762 & 0.508 & $\mathrm{R}$ & - & $86.40 \%$ \\
\hline & Caucasian & 1.02 & $0.88-1.18$ & 0.825 & 1 & 0.919 & $\mathrm{~F}$ & - & $0.00 \%$ \\
\hline \multirow[t]{4}{*}{ del/del vs. (ins/del+ins/ins) } & Overall & 0.93 & $0.71-1.21$ & 0.572 & 1 & 0.824 & $\mathrm{R}$ & 0.976 & $69.80 \%$ \\
\hline & Indian & 2.51 & 1.24-5.11 & 0.011 & 0.044 & 0.042 & $\mathbf{F}$ & - & $0.00 \%$ \\
\hline & East Asian & 0.56 & $0.36-0.86$ & 0.008 & 0.032 & 0.032 & $\mathbf{F}$ & - & $47.90 \%$ \\
\hline & Caucasian & 0.98 & $0.67-1.43$ & 0.919 & 1 & 0.919 & $\mathrm{R}$ & - & $78.10 \%$ \\
\hline \multirow[t]{4}{*}{ (del/del+ins/ins) vs. ins/del } & Overall & 1 & $0.91-1.09$ & 0.998 & 1 & 0.998 & $\mathrm{~F}$ & 0.899 & $0.00 \%$ \\
\hline & Indian & 0.96 & $0.72-1.29$ & 0.809 & 0.809 & 0.809 & $\mathrm{~F}$ & - & $0.00 \%$ \\
\hline & East Asian & 1.11 & $0.80-1.54$ & 0.538 & 0.762 & 0.538 & $\mathrm{R}$ & - & $68.20 \%$ \\
\hline & Caucasian & 0.99 & $0.86-1.13$ & 0.867 & 1 & 0.919 & $\mathrm{~F}$ & - & $44.90 \%$ \\
\hline \multirow[t]{4}{*}{ ins/del vs. del/del } & Overall & 1.06 & $0.83-1.36$ & 0.618 & 1 & 0.063 & $\mathrm{R}$ & 0.931 & $60.10 \%$ \\
\hline & Indian & 0.42 & $0.20-0.88$ & 0.021 & 0.063 & 0.042 & $\mathbf{F}$ & - & $0.00 \%$ \\
\hline & East Asian & 1.59 & $1.01-2.51$ & 0.047 & 0.141 & 0.094 & $\mathbf{F}$ & - & $0.00 \%$ \\
\hline & Caucasian & 1.03 & $0.70-1.51$ & 0.899 & 1 & 0.919 & $\mathrm{R}$ & - & $76.80 \%$ \\
\hline
\end{tabular}

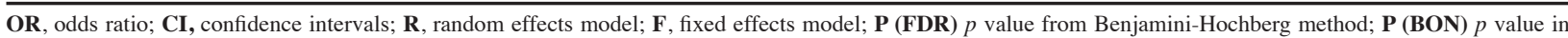
stepdown Bonferroni testing; The values given in bold represent statistically significant results

Table 4. Meta-analysis of The Casp8 D302H Polymorphism On PCa Risk

\begin{tabular}{|c|c|c|c|c|c|c|c|c|c|}
\hline \multirow[t]{2}{*}{ Comparison } & \multirow[t]{2}{*}{ Population } & \multicolumn{5}{|c|}{ Test of association } & \multirow[t]{2}{*}{ Mode } & \multirow{2}{*}{$\begin{array}{c}\text { Egger's test } \\
P\end{array}$} & \multirow{2}{*}{$\begin{array}{l}\text { Heterogeneity } \\
\qquad \mathrm{I}^{2}\end{array}$} \\
\hline & & OR & $95 \% \mathrm{CI}$ & $P$ & $P(\mathrm{BON})$ & $P(\mathrm{FDR})$ & & & \\
\hline \multirow[t]{4}{*}{$(\mathrm{DH}+\mathrm{HH})$ vs. $\mathrm{DD}$} & Overall & 0.77 & $0.47-1.28$ & 0.317 & 0.705 & 0.317 & $\mathrm{R}$ & 0.97 & $73.60 \%$ \\
\hline & Overall & $0.76^{*}$ & $0.63-0.91$ & 0.003 & - & - & $\mathrm{F}$ & - & $73.60 \%$ \\
\hline & Indian & 1.51 & $0.96-2.38$ & 0.077 & 0.308 & 0.193 & - & - & - \\
\hline & Non-Indian & 0.66 & $0.53-0.81$ & $<0.001$ & 0.002 & 0.001 & $\mathbf{F}$ & - & $0 \%$ \\
\hline \multirow[t]{3}{*}{$\mathrm{HH}$ vs. (DD+DH) } & Overall & 1.17 & $0.90-1.52$ & 0.249 & 0.705 & 0.317 & $\mathrm{~F}$ & 0.892 & $45.80 \%$ \\
\hline & Indian & 1.9 & $0.53-1.83$ & 0.335 & 0.354 & 0.335 & - & - & - \\
\hline & Non-Indian & 0.97 & $0.42-2.25$ & 0.952 & 0.952 & 0.952 & $\mathrm{R}$ & - & $60.20 \%$ \\
\hline \multirow[t]{4}{*}{$(\mathrm{DD}+\mathrm{HH})$ vs. $\mathrm{DH}$} & Overall & 1.27 & $0.92-1.75$ & 0.152 & 0.608 & 0.317 & $\mathrm{R}$ & 0.861 & $66.70 \%$ \\
\hline & Overall & $1.31 *$ & $1.11-1.54$ & 0.001 & - & - & $\mathrm{F}$ & 0.861 & $66.70 \%$ \\
\hline & Indian & 0.71 & $0.44-1.113$ & 0.145 & 0.354 & 0.193 & - & - & - \\
\hline & Non-Indian & 1.43 & $1.20-1.70$ & $<0.001$ & $<0.001$ & $<0.001$ & $\mathbf{F}$ & - & 0\% \\
\hline \multirow[t]{3}{*}{ DH vs. DD } & Overall & 0.73 & $0.44-1.22$ & 0.235 & 0.705 & 0.317 & $\mathrm{R}$ & 0.856 & $72.70 \%$ \\
\hline & Indian & 1.46 & $0.91-2.34$ & 0.118 & 0.354 & 0.193 & - & - & - \\
\hline & Non-Indian & 0.66 & $0.53-0.82$ & $<0.001$ & $<0.001$ & $<0.001$ & $\mathbf{F}$ & - & $9.60 \%$ \\
\hline
\end{tabular}

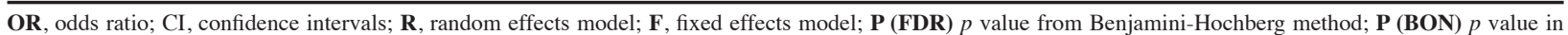
stepdown Bonferroni testing; The values given in bold represent statistically significant results; the values marked with a $*$ represent the results of the alternative approach (fixed effects despite heterogeneity)

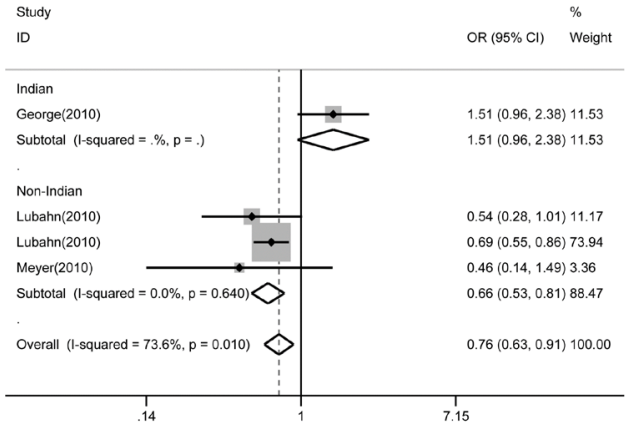

Figure 3. Forest Plot of Odd Ratios (ORs) of D302H Polymorphism Dominant Model (DH+HH vs. DD) Associated with PCa Stratified by Ethnicity Using a Fixed-Effect Mode

over-dominant (DD+HH vs. DH) genetic models. On the other hand, subgroup analysis stratified by ethnicity demonstrated that the $\mathrm{D} 302 \mathrm{H}$ polymorphism was a highly protective allele for $\mathrm{PCa}$ in the Non-Indian population in the dominant model $(\mathrm{OR}=0.66 ; 95 \% \mathrm{CI}=0.53-0.81$; $P<0.001 ; P_{b o n}=0.02 ; P_{f d r}=0.01$; Figure 4$)$ and overdominant model $(\mathrm{OR}=1.43 ; 95 \% \mathrm{CI}=1.20-1.70 ; P<0.001$; $P_{b o n}<0.001 ; P_{f d r}<0.001 ;$ Figure 4). The detailed data are

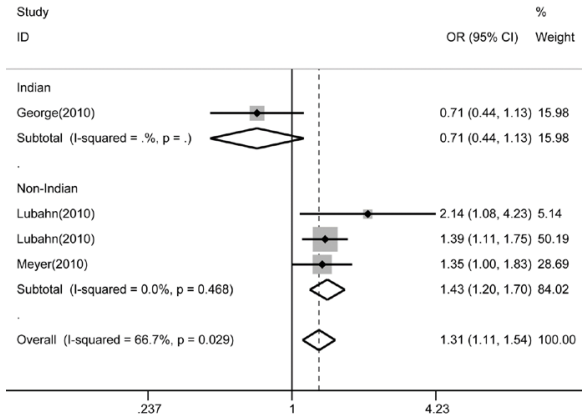

Figure 4. Forest Plot of Odd Ratios (ORs) of D302H Polymorphism Over-Dominant Model (DD+HH vs. DH) Associated with PCa Stratified by Ethnicity Using a Fixed-Effect Mode

presented in Table 4.

Sensitivity analysis

Because eligible studies were not available for sensitivity analysis of the $\mathrm{D} 302 \mathrm{H}$ polymorphism, a oneway sensitivity analysis across all ethnic groups was performed to evaluate the stability of the $-6526 \mathrm{~N}$ del polymorphism data. The results of this analysis showed 


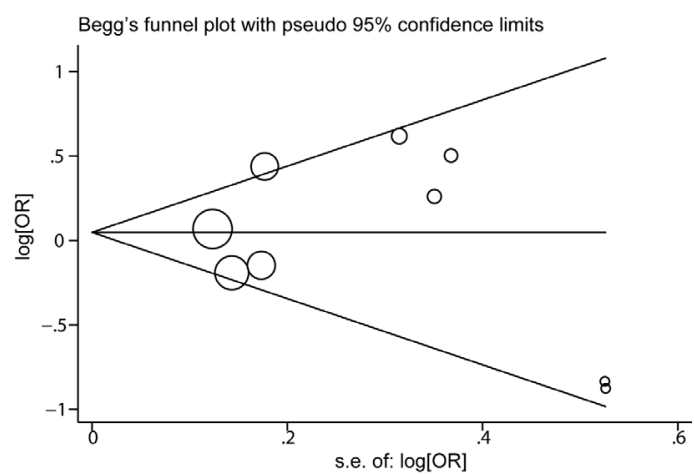

Figure 5. Begg's Funnel Plot for Publication Bias Test of $-6526 \mathrm{~N}$ del (ins/del $v s$. del/del)

that statistical significance was not changed when any single study was omitted (data not shown). The results of the sensitivity analysis demonstrated that the $-6526 \mathrm{~N}$ del polymorphism data in this meta-analysis were relatively stable and credible.

\section{Publication bias}

In the $-6526 \mathrm{~N}$ studies, both the Begg's funnel plot (Figure 5) and Egger's test were performed to estimate publication bias. Because of the limited number of $\mathrm{D} 302 \mathrm{H}$ studies, only Egger's test was performed. The results did not show any statistical evidence of publication bias (all $P>0.05)$. Results of the publication bias test are shown in Table 3.

\section{Discussion}

To date, our study is the first meta-analysis to determine the association of CASP8 $-6526 \mathrm{~N}$ del and D302H SNPs with PCa. The association of CASP8 $-6526 \mathrm{~N}$ del and D302H SNPs with PCa risk was evaluated in 8196 and 4201 subjects, respectively. The lack of significant results in the pooled analysis suggested the absence of robust associations between the 2 SNPs and PCa. In the pooled analysis, both SNPs showed substantial heterogeneity in all the genetic models. The subgroup analyses based on ethnicity yielded more homogeneous genetic effects than the pooled analyses. The sensitivity analysis of the $-6526 \mathrm{~N}$ del polymorphism indicated that our results were relatively robust.

Nowadays a number of studies have investigated association between CASP8 gene polymorphisms and risk of cancer. It is reported that CASP8 $-6526 \mathrm{~N}$ del and $\mathrm{D} 302 \mathrm{H}$ polymorphisms play a vital role in diversity tumors, such as lung cancer (Son et al., 2006), breast cancer (Cybulski. et al., 2007; Frank et al., 2008) and colorectal cancer (Wu et al., 2013). Sun et al. reported that the del allele of $-6526 \mathrm{~N}$ del reduces $\mathrm{T}$ lymphocyte apoptosis by inhibiting the binding site for the transcriptional activator stimulatory protein 1 in the CASP8 promoter (Sun et al., 2007). This function of $-6526 \mathrm{~N}$ del has been associated with increased immune surveillance (Sun et al., 2007). Therefore, individuals with the del allele may be less susceptible to tumorigenesis. However, different ethnicities may have distinct genetic backgrounds; therefore, tumor susceptibility can be influenced by ethnicity (Hirschhorn et al., 2002). The subgroup analyses revealed that the association of $-6526 \mathrm{~N}$ del with PCa susceptibility was paradoxical among the different ethnicities. The subgroup analysis stratified by ethnicity indicated that $-6526 \mathrm{~N}$ del was associated with increased PCa risk in subjects of Indian descent and with reduced $\mathrm{PCa}$ risk in subjects of East Asian descent. The $-6526 \mathrm{~N}$ del polymorphism was not significantly associated with PCa susceptibility in subjects of Caucasian descent under any genetic model. With regard to $\mathrm{D} 302 \mathrm{H}$ polymorphism, its functional effect is yet unknown. Between mouse and human, D302H map to an evolutionarily conserved region. This suggests that this variant can directly influence PCa rather than depending on an unknown linkage disequilibrium causative variant (Bethke et al., 2009). It has been suggested that the D302H polymorphism may affect the interaction of CASP8 with regulatory proteins (MacPherson et al., 2004; Bethke et al., 2009). Previous meta-analyses of the association of the $\mathrm{D} 302 \mathrm{H}$ polymorphism with cancer risk have been inconclusive (Bethke et al., 2009; Sergentanis and Economopoulos, 2010). Meanwhile, Michailidou (Michailidou et al., 2013) and Turnbull (Turnbull et al., 2010) indicated that D302H variant in CASP8 may be a false positive association through recent GWAS studies. In the present meta-analysis, the protective effect of the D302H polymorphism for PCa risk was observed only in the Non-Indian population under over-dominant, heterogeneous co-dominant, and dominant models.

Given the complex etiology of cancer, PCa risk cannot be attributed to a single factor. Demographic patterns, lifestyle, hormonal influences, inflammation, Statin drugs, cholesterol, and genome wide variation play a role in prostate carcinogenesis. Considering the multitude of factors associated with PCa risk, the varied results of CASP8 polymorphisms and PCa risk among the different ethnicities in our meta-analysis is not surprising. It's worth noting that the rare allele of $-6526 \mathrm{~N}$ del and D302H of CASP8 all have a trend of an increased risk of $\mathrm{PCa}$ in the Indian population. Meanwhile the rare allele of $-6526 \mathrm{~N}$ del has a positive association with $\mathrm{PCa}$ in the Indian population, but a negative observation in the East Asian population. Previous studies on genetic association suggested that the characteristics of Indian population might be different from other Asian populations. A metaanalysis of p53 codon 72 polymorphism conducted by Zhou et al. indicated that Pro carrier showed significant associations with increased risk of cervical cancer among Indian populations, but not among Chinese, Japanese and Korean populations (Zhou et al., 2012). However, the study on KCNQ1 rs2237892 C $\rightarrow$ T gene polymorphism and type 2 diabetes mellitus in the Asian population demonstrated that a significant association was found in Chinese, Korean and Malaysia population, but not in Indian population ( $\mathrm{Li}$ et al., 2014). The prevalence of prostate cancer and the allele frequencies differ across populations (Zhao et al., 2013), and some genes are specific in region and race, any conclusion concerning genetic effects should be carefully interpreted.

The limitations of our meta-analysis should be noted. First, although $9-6526 \mathrm{~N}$ del studies were included in 
the meta-analysis, the subgroup analysis was limited to 3 studies after stratification by ethnicity. The limited number of D302H studies in the subgroup analysis may have increased the risk of bias in the meta-analysis. Second, given that $\mathrm{PCa}$ is a multifactorial disease, confounding factors, such as age, tobacco smoking, PSA levels, clinical aggressiveness, and Gleason score, may have affected the associations between the 2 CASP8 polymorphisms and PCa risk. Finally, our analysis was based on unadjusted estimates. Adjusted ORs for the above risk factors should also be pooled to provide exact summary estimates. We were unable to perform these analyses because of the lack of individual data.

In conclusion, our meta-analysis demonstrated that the association of CASP8 $-6526 \mathrm{~N}$ del and D302H polymorphisms with $\mathrm{PCa}$ risk may be influenced by ethnicity. The association of the $-6526 \mathrm{~N}$ del polymorphism with PCa susceptibility was different among subjects of Asian descent. -652 6N del polymorphism was positively associated with $\mathrm{PCa}$ risk in the Indian population and negatively associated with PCa risk in the East Asian population. D302H polymorphism was associated with reduced PCa risk in the Non-Indian population. Given the limitations of our meta-analysis, confirmation of our findings in larger multicenter studies is warranted, especially between the Indian and East Asian populations.

\section{Acknowledgements}

This work was supported by National Natural Science Foundation of China (Grants number: 81060214, 81160097); the Research Fund for the Doctoral Program of Higher Education of China (Grants number: 20104503120008); the Guangxi Natural Science Foundation of China (Grants number: 2011GXNSFA018175, 2013GXNSFGA019005). We would like to thank Editage for providing editorial assistance.

\section{References}

Benjamini Y, Hochberg Y (1995). Controlling the False Discovery Rate: A Practical and Powerful Approach to Multiple Testing. J Royal Statistical Society, 57, 11.

Bethke L, Sullivan K, Webb E, et al (2009). CASP8 D302H and meningioma risk: an analysis of five case-control series. Cancer Lett, 273, 312-5.

Cybulski. C, Wokołorczyk. D, Gliniewicz. B (2007). A sixnucleotide deletion in the CASP8 promoter is not associated with a susceptibility to breast and prostate cancers in the Polish population. Breast Cancer Res Treat, 112, 367-368.

DerSimonian R, Laird N (1986). Meta-analysis in clinical trials. Control Clin Trials, 7, 177-88.

Frank B, Rigas SH, Bermejo JL, et al (2008). The CASP8 -652 $6 \mathrm{~N}$ del promoter polymorphism and breast cancer risk: a multicenter study. Breast Cancer Res Treat, 111, 139-44.

Fu G, Tang J, Wang M, et al (2011). CASP8 promoter polymorphism, mRNA expression and risk of prostate cancer among Chinese men. J Biomed Res, 25, 128-34.

George GP, Mandal RK, Kesarwani P, et al (2010). Polymorphisms and haplotypes in caspases 8 and 9 genes and risk for prostate cancer: a case-control study in cohort of North India. Urol Oncol, 30, 781-9.
Haiman CA, Garcia RR, Kolonel LN, et al (2008). A promoter polymorphism in the CASP8 gene is not associated with cancer risk. Nat Genet, 40, 259-60.

Higgins JP, Thompson SG, Deeks JJ, et al (2003). Measuring inconsistency in meta-analyses. $B M J, 327,557-60$.

Hirschhorn JN, Lohmueller K, Byrne E, et al (2002). A comprehensive review of genetic association studies. Genet Med, 4, 45-61.

Ho PK, Hawkins CJ (2005). Mammalian initiator apoptotic caspases. FEBS J, 272, 5436-53.

Holm S (1979). A simple sequentially rejective multiple test procedure. Scandinavian J Statistics, 6, 65-70.

Kesarwani P, Mandal RK, Maheshwari R, et al (2011). Influence of caspases 8 and 9 gene promoter polymorphism on prostate cancer susceptibility and early development of hormone refractory prostate cancer. BJU Int, 107, 471-6.

Klein LT, Lowe FC (1997). The effects of prostatic manipulation on prostate-specific antigen levels. Urol Clin North Am, 24, 293-7.

Li YY, Wang XM, Lu XZ (2014). KCNQ1 rs2237892 C-->T gene polymorphism and type 2 diabetes mellitus in the Asian population: a meta-analysis of 15,736 patients. J Cell Mol Med, 18, 274-82.

Lubahn J, Berndt SI, Jin CH, et al (2010). Association of CASP8 D302H polymorphism with reduced risk of aggressive prostate carcinoma. Prostate, 70, 646-53.

MacPherson G, Healey CS, Teare MD, et al (2004). Association of a common variant of the CASP8 gene with reduced risk of breast cancer. J Natl Cancer Inst, 96, 1866-9.

Mantel N, Haenszel W (1959). Statistical aspects of the analysis of data from retrospective studies of disease. J Natl Cancer Inst, 22, 719-48.

Meyer A, Coinac I, Bogdanova N, et al (2013). Apoptosis gene polymorphisms and risk of prostate cancer: a hospital-based study of German patients treated with brachytherapy. Urol Oncol, 31, 74-81.

Michailidou K, Hall P, Gonzalez-Neira A, et al (2013). Largescale genotyping identifies 41 new loci associated with breast cancer risk. Nat Genet, 45, 353-61.

Sergentanis TN, Economopoulos KP (2010). Association of two CASP8 polymorphisms with breast cancer risk: a metaanalysis. Breast Cancer Res Treat, 120, 229-34.

Siegel R, Naishadham D, Jemal A (2012). Cancer statistics, 2012. CA Cancer J Clin, 62, 10-29.

Son JW, Kang HK, Chae MH, et al (2006). Polymorphisms in the caspase-8 gene and the risk of lung cancer. Cancer Genet Cytogenet, 169, 121-7.

Sun T, Gao Y, Tan W, et al (2007). A six-nucleotide insertiondeletion polymorphism in the CASP8 promoter is associated with susceptibility to multiple cancers. Nat Genet, 39, 605-13.

Turnbull C, Ahmed S, Morrison J, et al (2010). Genomewide association study identifies five new breast cancer susceptibility loci. Nat Genet, 42, 504-7.

Wu Z, Li Y, Li S, et al (2013). Association between main Caspase gene polymorphisms and the susceptibility and prognosis of colorectal cancer. Med Oncol, 30, 565.

Zhao CX, Liu M, Wang JY, et al (2013). Association of 8 loci on chromosome 8q24 with prostate carcinoma risk in northern Chinese men. Asian Pac J Cancer Prev, 14, 6733-8.

Zheng SL, Sun J, Wiklund F, et al (2008). Cumulative association of five genetic variants with prostate cancer. $N$ Engl J Med, 358, 910-9.

Zhou X, Gu Y, Zhang SL (2012). Association between p53 codon 72 polymorphism and cervical cancer risk among Asians: a HuGE review and meta-analysis. Asian Pac J Cancer Prev, 13, 4909-14. 